\title{
Analytic capacity, rectifiability, and the Cauchy integral
}

\author{
Xavier Tolsa*
}

\begin{abstract}
A compact set $E \subset \mathbb{C}$ is said to be removable for bounded analytic functions if for any open set $\Omega$ containing $E$, every bounded function analytic on $\Omega \backslash E$ has an analytic extension to $\Omega$. Analytic capacity is a notion that, in a sense, measures the size of a set as a non removable singularity. In particular, a compact set is removable if and only if its analytic capacity vanishes. The so called Painlevé problem consists in characterizing removable sets in geometric terms. Recently many results in connection with this very old and challenging problem have been obtained. Moreover, it has also been proved that analytic capacity is semiadditive. We review these results and other related questions dealing with rectifiability, the Cauchy transform, and the Riesz transforms.
\end{abstract}

Mathematics Subject Classification (2000). Primary 30C85; Secondary 42B20, $28 \mathrm{~A} 75$.

Keywords. Analytic capacity, rectifiability, Cauchy transform, Riesz transform, singular integrals.

\section{Introduction}

In this paper we survey recent results in connection with analytic capacity, rectifiability and the Cauchy and Riesz transforms. We are specially interested in the interaction between analytic and geometric notions. Most of the results that we will review are a mixture of harmonic analysis and geometric measure theory. Some of them may have also some little amount of complex analysis.

Let us introduce some notation and definitions. A compact set $E \subset \mathbb{C}$ is said to be removable for bounded analytic functions if for any open set $\Omega$ containing $E$, every bounded function analytic on $\Omega \backslash E$ has an analytic extension to $\Omega$. In order to study removability, in the 1940's Ahlfors [Ah] introduced the notion of analytic capacity. The analytic capacity of a compact set $E \subset \mathbb{C}$ is

$$
\gamma(E)=\sup \left|f^{\prime}(\infty)\right|
$$

where the supremum is taken over all analytic functions $f: \mathbb{C} \backslash E \longrightarrow \mathbb{C}$ with $|f| \leq 1$ on $\mathbb{C} \backslash E$, and $f^{\prime}(\infty)=\lim _{z \rightarrow \infty} z(f(z)-f(\infty))$.

*Partially supported by grants MTM2004-00519 and Acción Integrada HF2004-0208 (Spain), and 2001-SGR-00431 (Generalitat de Catalunya). 
In [Ah], Ahlfors showed that $E$ is removable for bounded analytic functions if and only if $\gamma(E)=0$.

Painlevé's problem consists in characterizing removable singularities for bounded analytic functions in a metric/geometric way. By Ahlfors' result this turns out to be equivalent to describing compact sets with positive analytic capacity in metric/geometric terms.

Vitushkin in the 1950's and 1960's showed that analytic capacity plays a central role in problems of uniform rational approximation on compact sets of the complex plane. Because of its applications to this type of problems he raised the question of the semiadditivity of $\gamma$. Namely, does there exist an absolute constant $C$ such that

$$
\gamma(E \cup F) \leq C(\gamma(E)+\gamma(F)) ?
$$

It has recently been proved [To5] that analytic capacity is indeed semiadditive. Moreover, a characterization of removable sets for bounded analytic functions in terms of the so called curvature of measures is also given in [To5]. In Section 2 of the present paper we will review these results and other recent advances in connection with analytic capacity and Painlevé's problem. We will describe some of the ideas involved in their proofs. In particular, we will see that $L^{2}$ estimates for the Cauchy transform play a prominent role in most of these results.

Recall that if $\nu$ is a finite complex Borel measure on $\mathbb{C}$, the Cauchy transform (or Cauchy integral) of $\nu$ is defined by

$$
\mathcal{C} \nu(z)=\int \frac{1}{\xi-z} d \nu(\xi) .
$$

Although the integral above is absolutely convergent a.e. with respect to Lebesgue measure, it does not make sense, in general, for $z \in \operatorname{supp}(\nu)$. This is the reason why one considers the truncated Cauchy transform of $\nu$, which is defined as

$$
\mathcal{C}_{\varepsilon} \nu(z)=\int_{|\xi-z|>\varepsilon} \frac{1}{\xi-z} d \nu(\xi),
$$

for any $\varepsilon>0$ and $z \in \mathbb{C}$.

In Section 3 we will survey several results about the $\mu$-almost everywhere (a.e.) existence of the principal value

$$
\text { p.v.C } \mu(z)=\lim _{\varepsilon \rightarrow 0} \mathcal{C}_{\varepsilon} \mu(z),
$$

where $\mu$ is some positive finite Borel measure on $\mathbb{C}$, and its relationship with rectifiability.

Section 4 deals with the natural generalization of analytic capacity to higher dimensions, the so called Lipschitz harmonic capacity. The role played by the Cauchy transform in connection with analytic capacity corresponds to the Riesz transforms in the case of Lipschitz harmonic capacity. See Section 4 for more details.

In the final section of the paper we recall some open problems related to analytic capacity, the Cauchy and Riesz transforms, and rectifiability. This is a rather short 
list which reflects our personal interests and it is not intended to be a complete account of open problems in the area.

Some comments about the notation used in the paper: as usual, the letter ' $C$ ' stands for an absolute constant which may change its value at different occurrences. The notation $A \lesssim B$ means that there is a positive absolute constant $C$ such that $A \leq C B$. Also, $A \approx B$ is equivalent to $A \lesssim B \lesssim A$.

\section{Analytic capacity and the Cauchy transform}

2.1. Basic properties of analytic capacity. In a sense, analytic capacity measures the size of a set as a non removable singularity for bounded analytic functions. A direct consequence of the definition is that for all $\lambda \in \mathbb{C}$ and $E \subset \mathbb{C}$ compact one has $\gamma(\lambda+E)=\gamma(E)$ and $\gamma(\lambda E)=|\lambda| \gamma(E)$. Further, if $E$ is connected, then

$$
\operatorname{diam}(E) / 4 \leq \gamma(E) \leq \operatorname{diam}(E) .
$$

The second inequality (which holds for any compact set $E$ ) follows from the fact that the analytic capacity of a ball coincides with its radius, and the first one is a consequence of Koebe's 1/4 theorem (see [Gam, Chapter VIII] or [Gar2, Chapter I] for the details, for example). Thus if $E$ is connected and different from a point, then it is non removable. This implies that any removable compact set must be totally disconnected.

The relationship between analytic capacity and Hausdorff measure is the following:

- If $\operatorname{dim}_{H}(E)>1$ (here $\operatorname{dim}_{H}$ stands for the Hausdorff dimension), then $\gamma(E)>0$. This result is an easy consequence of Frostman's Lemma.

- $\gamma(E) \leq \mathcal{H}^{1}(E)$, where $\mathcal{H}^{s}$ is the $s$-dimensional Hausdorff measure, or length when $s=1$. This follows from Cauchy's integral formula, and it was proved by Painlevé about one hundred years ago. In particular, notice that if $\operatorname{dim}_{H}(E)<1$, then $\gamma(E)=0$.

By the statements above, one infers that dimension 1 is the critical dimension in connection with analytic capacity. It turns out that some sets of positive length and dimension 1 have positive analytic capacity (for example, a segment), while others have vanishing analytic capacity. The latter assertion was proved by Vitushkin [Vi1]. Later on, an easier example of a set with positive length and zero analytic capacity was found by Garnett [Gar1] and Ivanov [Iv].

2.2. The Cauchy transform and the capacity $\gamma_{+}$. Recall that given a positive Borel measure $\mu$ on the complex plane, $\mathcal{C} \mu$ stands for the Cauchy transform of $\mu$. If $f$ is a $\mu$-measurable function $f$ on $\mathbb{C}$, we denote $\mathcal{C}_{\mu} f(z):=\mathcal{C}(f d \mu)(z)$ for $z \notin \operatorname{supp}(f)$, and $\mathcal{C}_{\mu, \varepsilon} f(z):=\mathcal{C}_{\varepsilon}(f d \mu)(z)$ for any $\varepsilon>0$ and $z \in \mathbb{C}$. We say that $\mathcal{C}_{\mu}$ is bounded on $L^{2}(\mu)$ if the operators $\mathcal{C}_{\mu, \varepsilon}$ are bounded on $L^{2}(\mu)$ uniformly on $\varepsilon>0$. 
Let us denote by $M_{+}(\mathbb{C})$ the set of finite (positive) Borel measures on $\mathbb{C}$. The capacity $\gamma_{+}$of a compact set $E \subset \mathbb{C}$ is

$$
\gamma_{+}(E):=\sup \left\{\mu(E): \mu \in M_{+}(\mathbb{C}), \operatorname{supp}(\mu) \subset E,\|\mathcal{C} \mu\|_{L^{\infty}(\mathbb{C})} \leq 1\right\} .
$$

That is, $\gamma_{+}$is defined as $\gamma$ in (1) with the additional constraint that $f$ should coincide with $\mathcal{C} \mu$, where $\mu$ is some positive Borel measure supported on $E$ (observe that $(\mathcal{C} \mu)^{\prime}(\infty)=-\mu(\mathbb{C})$ for any Borel measure $\left.\mu\right)$. Moreover, there is another slight difference: in (1) we required $\|f\|_{L^{\infty}(\mathbb{C} \backslash E)} \leq 1$, while in $(2),\|f\|_{L^{\infty}(\mathbb{C})} \leq 1$ (for $f=\mathcal{C} \mu$ ). Trivially, we have $\gamma_{+}(E) \leq \gamma(E)$.

We introduce now some additional notation. A Borel measure $\mu$ on $\mathbb{R}^{d}$ has growth of degree $n$ if there exists some constant $C$ such that $\mu(B(x, r)) \leq C r^{n}$ for all $x \in \mathbb{R}^{d}, r>0$. When $n=1$, we say that $\mu$ has linear growth. If $\mu$ satisfies $\mu(B(x, r)) \approx r^{n}$ for all $x \in \operatorname{supp}(\mu), 0<r \leq \operatorname{diam}(\operatorname{supp}(\mu))$, we say that $\mu$ is $n$-Ahlfors-David (n-AD) regular, or abusing language, AD regular. A set $E \subset \mathbb{C}$ is called $n$-AD regular (abusing language, $\mathrm{AD}$ regular) if $\mathcal{H}_{\mid E}^{n}$ is $\mathrm{AD}$ regular. We say that $\mu$ is doubling if there exists some constant $C$ such that $\mu(B(z, 2 r)) \leq C \mu(B(z, r))$ for all $z \in \operatorname{supp}(\mu), r>0$. In particular, $A D$ regular measures are doubling.

Next theorem shows why $L^{2}$ estimates for the Cauchy transform are useful in connection with analytic capacity.

Theorem 2.1. Let $\mu$ be a measure with linear growth on $\mathbb{C}$. Suppose that the Cauchy transform is bounded in $L^{2}(\mu)$. Then, for any compact $E \subset \mathbb{C}$ there exists a function $h$ supported on $E$ with $0 \leq h \leq 1$ such that $\int h d \mu \approx \mu(E)$, with $\left\|\mathcal{C}_{\varepsilon}(h d \mu)\right\|_{\infty} \leq C$ for all $\varepsilon>0$, and $\|\mathcal{C}(h d \mu)\|_{\infty, \mathbb{C} \backslash E} \leq C$. All the constants depend only on the linear growth of $\mu$ and on $\|\mathcal{C}\|_{L^{2}(\mu), L^{2}(\mu)}$.

In the statement above, $\left\|\mathcal{C}_{\mu}\right\|_{L^{2}(\mu), L^{2}(\mu)}$ stands for the operator norm of $\mathcal{C}_{\mu}$ on $L^{2}(\mu)$. That is, $\left\|\mathcal{C}_{\mu}\right\|_{L^{2}(\mu), L^{2}(\mu)}=\sup _{\varepsilon>0}\left\|\mathcal{C}_{\mu, \varepsilon}\right\|_{L^{2}(\mu), L^{2}(\mu)}$.

From the preceding result one infers that if $E$ supports a non zero measure $\mu$ with linear growth such that the Cauchy transform $\mathcal{C}_{\mu}$ is bounded on $L^{2}(\mu)$, then $\gamma(E) \geq \gamma_{+}(E)>0$.

Theorem 2.1 is from Davie and Øksendal [DØ] and it follows from the fact the $L^{2}$ boundedness of the Cauchy transform implies weak $(1,1)$ estimates. Theorem 2.1 is obtained by a suitable dualization of these weak $(1,1)$ estimates. See [Uy] for a connected result prior to $[\mathrm{D} \varnothing]$ which also involves a dualization of a weak $(1,1)$ inequality.

2.3. Menger curvature and rectifiability. Given three pairwise different points $x, y, z \in \mathbb{C}$, their Menger curvature is

$$
c(x, y, z)=\frac{1}{R(x, y, z)},
$$

where $R(x, y, z)$ is the radius of the circumference passing through $x, y, z$. If two among these points coincide, we let $c(x, y, z)=0$. For a positive Borel measure $\mu$, 
we define the curvature of $\mu$ as

$$
c^{2}(\mu)=\iiint c(x, y, z)^{2} d \mu(x) d \mu(y) d \mu(z) .
$$

Given $\varepsilon>0, c_{\varepsilon}^{2}(\mu)$ stands for the $\varepsilon$-truncated version of $c^{2}(\mu)$, defined as in the right hand side of $(3)$, but with the triple integral over $\left\{(x, y, z) \in \mathbb{C}^{3}:|x-y|, \mid y-\right.$ $z|| x-z \mid,>\varepsilon\}$.

The notion of curvature of a measure was introduced by Melnikov [Me] when he was studying a discrete version of analytic capacity, and it is one of the notions which is responsible of the big recent advances in connection with analytic capacity. The notion of curvature is connected to the Cauchy transform by the following result, proved by Melnikov and Verdera $[\mathrm{MeV}]$.

Proposition 2.2. Let $\mu$ be a Borel measure on $\mathbb{C}$ with linear growth. We have

$$
\left\|\mathcal{C}_{\varepsilon} \mu\right\|_{L^{2}(\mu)}^{2}=\frac{1}{6} c_{\varepsilon}^{2}(\mu)+O(\mu(\mathbb{C}))
$$

where $|O(\mu(\mathbb{C}))| \leq C \mu(\mathbb{C})$.

Sketch of the proof of Proposition 2.2. If we don't worry about truncations and the absolute convergence of the integrals, we can write

$$
\|\mathcal{C} \mu\|_{L^{2}(\mu)}^{2}=\int\left|\int \frac{1}{y-x} d \mu(y)\right|^{2} d \mu(x)=\iiint \frac{1}{(y-x)(\overline{z-x})} d \mu(y) d \mu(z) d \mu(x) .
$$

By Fubini (assuming that it can be applied correctly), permuting $x, y, z$, we get,

$$
\|\mathcal{C} \mu\|_{L^{2}(\mu)}^{2}=\frac{1}{6} \iiint \sum_{s \in S_{3}} \frac{1}{\left(z_{s_{2}}-z_{s_{1}}\right)\left(\overline{z_{s_{3}}-z_{s_{1}}}\right)} d \mu\left(z_{1}\right) d \mu\left(z_{2}\right) d \mu\left(z_{3}\right)
$$

where $S_{3}$ is the group of permutations of three elements. An elementary calculation shows that

$$
\sum_{s \in S_{3}} \frac{1}{\left(z_{s_{2}}-z_{s_{1}}\right)\left(\overline{z_{s_{3}}-z_{s_{1}}}\right)}=c\left(z_{1}, z_{2}, z_{3}\right)^{2} .
$$

So we get

$$
\|\mathcal{C} \mu\|_{L^{2}(\mu)}^{2}=\frac{1}{6} c^{2}(\mu)
$$

To argue rigorously, above we should use the truncated Cauchy transform $\mathcal{C}_{\varepsilon} \mu$ instead of $\mathcal{C} \mu$, and then we would obtain

$$
\begin{aligned}
\left\|\mathcal{C}_{\varepsilon} \mu\right\|_{L^{2}(\mu)}^{2} & =\iiint_{\substack{|x-y|>\varepsilon \\
|x-z|>\varepsilon}} \frac{1}{(y-x)(\overline{z-x})} d \mu(y) d \mu(z) d \mu(x) \\
& =\iiint_{\substack{|x-y|>\varepsilon \\
|x-z|>\varepsilon \\
|y-z|>\varepsilon}} \frac{1}{(y-x)(\overline{z-x})} d \mu(y) d \mu(z) d \mu(x)+O(\mu(\mathbb{C})) .
\end{aligned}
$$

By the linear growth of $\mu$, it is easy to check that $|O(\mu(\mathbb{C}))| \leq \mu(\mathbb{C})$. As above, using Fubini and permuting $x, y, z$, one shows that the triple integral in (5) equals $c_{\varepsilon}^{2}(\mu) / 6$. 
The identity (4) is remarkable because it relates an analytic notion (the Cauchy transform of a measure) with a metric/geometric one (curvature).

The above proposition was used in $[\mathrm{MeV}]$ to give a simple geometric proof of the $L^{2}$ boundedness of the Cauchy transform on a Lipschitz graph (the original proof is from Coifman, McIntosh and Meyer [CMM]). Indeed, using a Fourier type estimate, it was proved in $[\mathrm{MeV}]$ that if $\Gamma$ is a Lipschitz graph, then $c^{2}\left(\mathcal{H}_{\Gamma \cap B(z, r)}^{1}\right) \lesssim$ $r$ for all $z \in \Gamma, r>0$. From (4) one infers that $\left\|\mathcal{C}_{\varepsilon}\left(b \chi_{B(z, r)} \mathcal{H}_{\mid \Gamma}^{1}\right)\right\|_{L^{2}\left(\mathcal{H}_{\mid \Gamma \cap B(z, r)}^{1}\right)} \lesssim$ $\|b\|_{\infty} r^{1 / 2}$ for all $b \in L^{\infty}, z \in \Gamma$, and $r>0$, uniformly on $\varepsilon>0$. Now a simple argument shows that $\mathcal{C}_{\mathcal{H}_{\mid \Gamma}^{1}}$ sends boundedly $L^{\infty}$ into $B M O\left(\mathcal{H}_{\mid \Gamma}^{1}\right)$ and thus $H^{1}\left(\mathcal{H}_{\mid \Gamma}^{1}\right)$ into $L^{1}\left(\mathcal{H}_{\mid \Gamma}^{1}\right)$. Interpolating one gets the conclusion.

Let us turn our attention to rectifiability and its relationship with curvature of measures. A set is called rectifiable if it is $\mathcal{H}^{1}$-almost all contained in a countable union of rectifiable curves. On the other hand, it is called purely unrectifiable if it intersects any rectifiable curve at most in a set of zero length.

Now we wish to recall the traveling salesman theorem of P. Jones [Jo]. First we introduce some notation. Given $E \subset \mathbb{C}$ and a square $Q$, let $V_{Q}$ be an infinite strip (or line in the degenerate case) of smallest possible width which contains $E \cap 3 Q$, and let $w\left(V_{Q}\right)$ denote the width of $V_{Q}$. Then we set

$$
\beta_{E}(Q)=\frac{w\left(V_{Q}\right)}{\ell(Q)}
$$

where $\ell(Q)$ stands for the side length of $Q$. We denote by $\mathcal{D}$ the family of all dyadic squares in $\mathbb{C}$. In $[\mathrm{Jo}]$ the following result was proved:

Theorem 2.3. A set $E \subset \mathbb{C}$ is contained in a rectifiable curve $\Gamma$ (with finite length) if and only

$$
\sum_{Q \in \mathcal{D}} \beta_{E}(Q)^{2} \ell(Q)<\infty
$$

Moreover, the length of the shortest curve $\Gamma$ containing E satisfies

$$
\mathcal{H}^{1}(\Gamma) \approx \operatorname{diam}(E)+\sum_{Q \in \mathcal{D}} \beta_{E}(Q)^{2} \ell(Q),
$$

with absolute constants.

The theorem also holds for sets $E$ contained in $\mathbb{R}^{d}$. The proof of the "if" part of the theorem in [Jo] is also valid in this case. The "only if" part (for $\mathbb{R}^{d}$ ) was proved by Okikiolu [Ok]. Several versions of Jones' result which involve $n$-dimensional AD regular sets in $\mathbb{R}^{d}$ have been obtained by David and Semmes [DS1], [DS2]. In fact, David and Semmes have developed a whole theory of the so called "uniform rectifiability" for $n$-dimensional AD regular sets in $\mathbb{R}^{d}$.

Next result, proved by $\mathrm{P}$. Jones (see [Pa, Chapter 3$]$ ), shows that there is a strong connection between curvature and the coefficients $\beta$ in Jones' traveling salesman theorem. 
Theorem 2.4. (a) If $E \subset \mathbb{C}$ is $1-A D$ regular, then

$$
\sum_{Q \in \mathcal{D}} \beta_{E}(Q)^{2} \ell(Q) \leq C c^{2}\left(\mathcal{H}_{\mid E}^{1}\right)
$$

where $C$ depends only on the $A D$ regularity constant of $E$.

(b) If $\mu$ is a measure with linear growth supported on a rectifiable curve $\Gamma \subset \mathbb{C}$, then

$$
c^{2}(\mu) \leq C \sum_{Q \in \mathcal{D}} \beta_{\Gamma}(Q)^{2} \mu(Q),
$$

where $C$ depends only on the linear growth constant of $\mu$.

From (a) in the preceding theorem and Theorem 2.3 it turns out that if $E \subset \mathbb{C}$ is $\mathrm{AD}$ regular and $c^{2}\left(\mathcal{H}_{\mid E}^{1}\right)<\infty$, then $E$ is rectifiable. If one does not assume $E$ to be AD regular, David and Léger [Lé] showed that the result still holds:

Theorem 2.5. Let $E \subset \mathbb{C}$ be compact with $\mathcal{H}^{1}(E)<\infty$. If $c^{2}\left(\mathcal{H}_{\mid E}^{1}\right)<\infty$, then $E$ is rectifiable.

The proof of this result in [Lé] uses geometric techniques, in the spirit of the ones used by P. Jones for Theorem 2.3 in [Jo] and by David and Semmes in [DS1]. Recently, in [To9] a very different proof of Theorem 2.5 has been obtained. The new arguments are based on some kind of isoperimetric inequality involving analytic capacity and on the characterization of rectifiability in terms of densities.

From Theorem 2.5 and Proposition 2.2 one infers that if $\mathcal{H}^{1}(E)<\infty$ and the Cauchy transform is bounded on $L^{2}\left(\mathcal{H}_{\mid E}^{1}\right)$, then $E$ must be rectifiable. A more quantitative version of this result proved by Mattila, Melnikov and Verdera [MMV] asserts that if $E$ is $\mathrm{AD}$ regular and the Cauchy transform is bounded on $L^{2}\left(\mathcal{H}_{\mid E}^{1}\right)$, then $E$ is contained in an $\mathrm{AD}$ regular curve $\Gamma$.

Recently, some of the results above dealing with rectifiability, $\beta$ 's, and curvature have been extended in different directions. For example, Lerman [Lr] has obtained a result analogous to Theorem 2.3 which involves very general Borel measures $\mu$ on $\mathbb{R}^{d}$ (instead of $\mathcal{H}_{\mid E}^{1}$ ) and $L^{2}(\mu)$ versions of Jones' $\beta$ 's. Ferrari, Franchi and Pajot [FFP] have extended the "if" part of the Theorem 2.3 to the Heisenberg group. Schul [Sch] has proved a version of the same theorem which is valid for Hilbert spaces. On the other hand, Hahlomaa [Hah] has obtained a version of Léger's Theorem 2.5 suitable for metric spaces.

2.4. Vitushkin's conjecture. For $\theta \in[0, \pi)$, let $p_{\theta}$ denote the orthogonal projection onto the line through the origin and direction $(\cos \theta, \sin \theta)$. Given a Borel set $E \subset \mathbb{C}$, its Favard length is

$$
\operatorname{Fav}(E)=\int_{0}^{\pi} \mathcal{H}^{1}\left(p_{\theta}(E)\right) d \theta
$$

Vitushkin conjectured in the 1960's that $\gamma(E)>0$ if and only if $\operatorname{Fav}(E)>0$. In 1986 Mattila [Ma1] showed that this conjecture is false. Indeed, he proved that 
the property of having positive Favard length is not invariant under conformal mappings while removability for bounded analytic functions remains invariant. Mattila's result didn't tell which implication in the above conjecture was false. Later on, Jones and Murai [JM] constructed a set with zero Favard length and positive analytic capacity. An easier example using curvature was obtained more recently by Joyce and Mörters $[\mathrm{JyM}]$.

Although Vitushkin's conjecture is not true in full generality, it turns out that it holds in the particular case of sets with finite length. This was proved by G. David [Da] in 1998. Recall that when has $E$ with finite length, by Besicovitch theorem, $\operatorname{Fav}(E)=0$ if and only if $E$ is purely unrectifiable.

The precise statement of David's result is the following.

Theorem 2.6. Let $E \subset \mathbb{C}$ be compact with $\mathcal{H}^{1}(E)<\infty$. Then, $\gamma(E)=0$ if and only if $E$ is purely unrectifiable.

This result is the solution of Painlevé's problem for sets with finite length. To be precise, let us remark that the "if" part of the theorem is not due to David. In fact, it follows from Calderón's theorem on the $L^{2}$ boundedness of the Cauchy transform on Lipschitz graphs with small Lipschitz constant and from Theorem 2.1. The "only if" part of the theorem, which is more difficult, is the one proved by David. Let us also mention that Mattila, Melnikov and Verdera [MMV] had proved previously the same result under the assumption that $E$ is a 1-dimensional AD regular set.

The scheme of the proof of the "only if" part of Theorem 2.6 is the following. Let $E \subset \mathbb{C}$ be compact with $\gamma(E)>0$ and finite length. Then there exists a function $f$ analytic on $\mathbb{C} \backslash E$ such that $|f(z)| \leq 1$ on $\mathbb{C} \backslash E$ and $f^{\prime}(\infty)=\gamma(E)$ (this is the so called Ahlfors function, which maximizes $\gamma(E)$ ). Since $\mathcal{H}^{1}(E)<$ $\infty$ it is not difficult to see that there exists some complex, bounded function $g$ supported on $E$ such that $f(z)=\mathcal{C}\left(g d \mathcal{H}_{\mid E}^{1}\right)(z)$ for $z \notin E$. Then it easily follows that $\left\|\mathcal{C}_{\varepsilon}\left(g d \mathcal{H}_{\mid E}^{1}\right)\right\|_{L^{\infty}} \leq C$ uniformly on $\varepsilon>0$. On the other hand, $g$ also satisfies $\left|\int g d \mathcal{H}_{\mid E}^{1}\right|=\left|f^{\prime}(\infty)\right|=\gamma(E)>0$. By a suitable $T(b)$ type theorem (which involves some delicate stopping time arguments, and non doubling measures) proved in [Da], one infers that there exists a subset $F \subset E$ with $\mathcal{H}^{1}(F)>0$ such the Cauchy transform is bounded on $L^{2}\left(\mathcal{H}_{\mid F}^{1}\right)$. From Proposition 2.2 it follows that $c^{2}\left(\mathcal{H}_{\mid F}^{1}\right)<\infty$, and then by Theorem $2.5 F$ is rectifiable. So $E$ cannot be purely unrectifiable.

Let us remark that the $T(b)$ theorem in [Da] uses a preliminary result from $[\mathrm{DM}]$. A similar theorem had been previously obtained by Christ $[\mathrm{Ch}]$ in the AD regular case.

The result analogous to Theorem 2.6 for sets with infinite length is false. For this type of sets there is no such a nice geometric solution of Painlevé's problem, and we have to content ourselves with a characterization such as the one in Corollary 2.10 below (at least, for the moment).

2.5. Characterization of $\gamma_{+}$in terms of curvature of measures and $L^{2}$ estimates for the Cauchy transform. The following theorem 
characterizes $\gamma_{+}$in terms of curvature of measures and in terms of the $L^{2}$ norm of the Cauchy transform.

Theorem 2.7. Let $\Sigma(E)$ denote class of Borel measures supported on $E$ such that $\mu(B(x, r)) \leq r$ for all $x \in \mathbb{C}, r>0$. For any compact set $E \subset \mathbb{C}$ we have

$$
\begin{aligned}
\gamma_{+}(E) & \approx \sup \left\{\mu(E): \mu \in \Sigma(E), c^{2}(\mu) \leq \mu(E)\right\} \\
& \approx \sup \left\{\mu(E): \mu \in \Sigma(E),\left\|\mathcal{C}_{\mu}\right\|_{L^{2}(\mu), L^{2}(\mu)} \leq 1\right\}
\end{aligned}
$$

Sketch of the proof of Theorem 2.7. Call $S_{1}$ and $S_{2}$ the first and second suprema on the right side of $(8)$, respectively.

To see that $S_{1} \gtrsim \gamma_{+}(E)$ take $\mu$ supported on $E$ such that $\|\mathcal{C} \mu\|_{\infty} \leq 1$ and $\mu(E) \geq \gamma_{+}(E) / 2$. One easily gets that $\left\|\mathcal{C}_{\varepsilon} \mu\right\|_{\infty} \lesssim 1$ on $\operatorname{supp}(\mu)$ for every $\varepsilon>0$ and $\mu(B(x, r)) \leq C r$ for all $r>0$. From Proposition 2.2, it follows then that $c^{2}(\mu) \leq C \mu(E)$.

Consider now the inequality $S_{2} \gtrsim S_{1}$. Let $\mu$ be supported on $E$ with linear growth such that $c^{2}(\mu) \leq \mu(E)$ and $\widetilde{S}_{1} \leq 2 \mu(E)$. We set

$$
A:=\left\{x \in E: \iint c(x, y, z)^{2} d \mu(y) d \mu(z) \leq 2\right\}
$$

By Tchebychev $\mu(A) \geq \mu(E) / 2$. Moreover, for any set $B \subset \mathbb{C}$,

$$
c^{2}\left(\mu_{\mid B \cap A}\right) \leq \iiint_{x \in B \cap A} c(x, y, z)^{2} d \mu(x) d \mu(y) d \mu(z) \leq 2 \mu(B) .
$$

In particular, this estimate holds when $B$ is any square in $\mathbb{C}$. Then, by the so called $T(1)$ theorem (see [To1] or [NTV1]), one infers that $\mathcal{C}_{\mu_{\mid A}}$ is bounded on $L^{2}\left(\mu_{\mid A}\right)$. Thus $S_{2} \gtrsim \mu(A) \approx S_{1}$.

Finally, the inequality $\gamma_{+}(E) \gtrsim S_{2}$ follows from Theorem 2.1.

For the complete arguments of the preceding proof, see [To1] or [To4]. Notice that since the term

$$
\sup \left\{\mu(E): \mu \in \Sigma(E),\left\|\mathcal{C}_{\mu}\right\|_{L^{2}(\mu), L^{2}(\mu)} \leq 1\right\}
$$

is countably semiadditive, from Theorem 2.7 one infers that $\gamma_{+}$is also countably semiadditive.

Corollary 2.8. The capacity $\gamma_{+}$is countably semiadditive. That is, if $E_{i}, i=$ $1,2, \ldots$, is a countable (or finite) family of compact sets, we have

$$
\gamma_{+}\left(\bigcup_{i=1}^{\infty} E_{i}\right) \leq C \sum_{i=1}^{\infty} \gamma_{+}\left(E_{i}\right)
$$


2.6. Comparability between $\gamma$ and $\gamma_{+}$. In [To5] the following result has been proved.

Theorem 2.9. There exists an absolute constant $C$ such that for any compact set $E \subset \mathbb{C}$ we have

$$
\gamma(E) \leq C \gamma_{+}(E) .
$$

As a consequence, $\gamma(E) \approx \gamma_{+}(E)$.

The comparability between $\gamma$ and $\gamma_{+}$had been previously proved by P. Jones for compact connected sets by geometric arguments, very different from the ones in [To5] (see [Pa, Chapter 3]). Also, in [MTV] it had already been shown that $\gamma \approx \gamma_{+}$holds for a big class of Cantor sets. The proof of Theorem 2.9 in [To5] is inspired in part by the ideas in [MTV].

An obvious corollary of Theorem 2.9 and the characterization of $\gamma_{+}$in terms of curvature in Theorem 2.7 is the following.

Corollary 2.10. Let $E \subset \mathbb{C}$ be compact. Then, $\gamma(E)>0$ if and only if $E$ supports a non zero Borel measure with linear growth and finite curvature.

Since we know that $\gamma_{+}$is countably semiadditive, the same happens with $\gamma$ :

Corollary 2.11. Analytic capacity is countably semiadditive. That is, if $E_{i}, i=$ $1,2, \ldots$, is a countable (or finite) family of compact sets, we have

$$
\gamma\left(\bigcup_{i=1}^{\infty} E_{i}\right) \leq C \sum_{i=1}^{\infty} \gamma\left(E_{i}\right)
$$

Some few words about the proof of Theorem 2.7: it is enough to show that there exists some measure $\mu$ supported on $E$ with linear growth, satisfying $\mu(E) \approx$ $\gamma(E)$, and such that the Cauchy transform $\mathcal{C}_{\mu}$ is bounded on $L^{2}(\mu)$ with absolute constants. To this end, an important tool used in [To5] is the $T(b)$ theorem of Nazarov, Treil and Volberg in [NTV2], which is valid for non doubling measures. To apply this $T(b)$ theorem, one has to construct a suitable measure $\mu$ and a function $g \in L^{\infty}(\mu)$ fulfilling some precise conditions, similarly to the proof of Vitushkin's conjecture by David.

However, the situation now is more delicate because a direct application of that $T(b)$ theorem does not suffice. Indeed, let $f$ be the Ahlfors function of $E$, so that $f$ is analytic and bounded in $\mathbb{C} \backslash E$, with $f^{\prime}(\infty)=\gamma(E)$. By a standard approximation argument, it is not difficult to see that one can assume that $\mathcal{H}^{1}(E)<\infty$. Thus there exists some function $g$ such that $f(z)=\mathcal{C}\left(g d \mathcal{H}_{\mid E}^{1}\right)(z)$ for $z \notin E$. If we argue like in [Da] (see Subsection 2.4 above), we will deduce that there exists a subset $F \subset E$ such that the Cauchy transform is bounded in $L^{2}\left(\mathcal{H}_{\mid F}^{1}\right)$. However, the size of $F$ and the $L^{2}$ norm of the Cauchy transform $\mathcal{C}_{\mathcal{H}_{\mid F}^{1}}$ will depend strongly on the ratio $\mathcal{H}^{1}(E) / \gamma(E)$, which blows up as $\mathcal{H}^{1}(E) \rightarrow \infty$ or $\gamma(E) \rightarrow 0$. This difficulty is overcome in [To5] by using some ideas from potential theory and a suitable "induction on scales" argument. 
Corollary 2.10 yields a characterization of removable sets for bounded analytic functions in terms of curvature of measures. Although this result has a definite geometric flavor, it is not clear if this is a really good geometric characterization. Nevertheless, in [To7] it has been shown that the characterization is invariant under bilipschitz mappings, using a corona type decomposition for non doubling measures. Previously, Garnett and Verdera [GV] had proved an analogous result for some Cantor sets. The problem about the behavior of removability and analytic capacity under bilipschitz mappings was raised by Verdera. See [Ve2].

2.7. Other results. In [To6], some results analogous to Theorems 2.7 and 2.9 have been obtained for the continuous analytic capacity $\alpha$. This capacity is defined like $\gamma$ in (1), with the additional requirement that the functions $f$ considered in the sup should extend continuously to the whole complex plane. The capacity $\alpha$ is important because its many applications in connection with problems of uniform rational approximation in the complex plane, as shown by Vitushkin [Vi3]. In [To6] it is proved that $\alpha$ is semiadditive. This result has some nice consequences. For example, it implies the so called inner boundary conjecture.

The inner boundary of a compact set $E \subset \mathbb{C}$, denoted by $\partial_{i} E$, is the set of boundary points of $E$ which do not belong to the boundary of any connected component of $\mathbb{C} \backslash E$. The inner boundary conjecture (or theorem) says that if $\alpha\left(\partial_{i} E\right)=0$, then any function analytic in $\stackrel{\circ}{E}$ and continuous on $E$ can be approximated uniformly by functions which are analytic in neighborhoods of $E$ (i.e. different neighborhoods for different functions).

The techniques for the proof of Theorem 2.9 have also been used by Prat [Pr] and Mateu, Prat and Verdera $[\mathrm{MPV}]$ to study the capacities $\gamma_{s}$ associated to the $s$-dimensional signed Riesz kernel $k_{s}(x)=x /|x|^{s+1}$, with $s$ non integer. Given a compact $E \subset \mathbb{R}^{n}$, the precise definition of $\gamma_{s}(E)$ is

$$
\gamma_{s}(E)=\sup |\langle\nu, 1\rangle|,
$$

where the supremum is taken over all distributions $\nu$ supported on $E$ such that $K_{s} * \nu$ is an $L^{\infty}$ function with $\left\|K_{s} * \nu\right\|_{\infty} \leq 1$.

The results in $[\mathrm{Pr}]$ and $[\mathrm{MPV}]$ show that the behavior of $\gamma_{s}$ with $s$ non integer is very different from the one with $s$ integer. In $[\mathrm{Pr}]$ it is shown that sets with finite $s$-dimensional Hausdorff measure have vanishing capacity $\gamma_{s}$ when $0<s<$ 1. Moreover, for these $s$ 's it is proved in $[\mathrm{MPV}]$ that $\gamma_{s}$ is comparable to the capacity $C_{\frac{2}{3}(n-s), \frac{3}{2}}$ from nonlinear potential theory. Recall that for $1<p<\infty$ and $0<t p \leq d$ the capacity $C_{t, p}$ of a compact set $E \subset \mathbb{R}^{d}$ is defined by

$$
C_{t, p}(E)=\inf \left\{\|\varphi\|_{p}^{p}: \varphi \in \mathcal{C}_{c}^{\infty}\left(\mathbb{R}^{d}\right), \varphi * \frac{1}{|x|^{d-t}} \geq 1 \text { on } E\right\} .
$$

It is not known if the comparability $\gamma_{s} \approx C_{\frac{2}{3}(n-s), \frac{3}{2}}$ holds for non integers $s>1$. This case seems much more difficult to study, although in the AD regular situation some results have been obtained in $[\mathrm{Pr}]$. 
Using the corona type decomposition for measures with finite curvature and linear growth obtained in [To7], it has been proved in [To8] that if $\mu$ is a measure without atoms such that the Cauchy transform is bounded on $L^{2}(\mu)$, then any Calderón-Zygmund operator associated to an odd kernel sufficiently smooth is also bounded in $L^{2}(\mu)$.

\section{Principal values for the Cauchy integral and re- lated results}

There is a strong relationship between rectifiability and the behavior of the Cauchy transform. Indeed, in Section 2 we saw that the $L^{2}$ boundedness of the Cauchy transform with respect to the arc length measure implies rectifiability. In this section we will describe some related results which involve the existence of principal values instead of $L^{2}$ boundedness.

Recall that given a Borel measure $\mu$ on $\mathbb{C}$, the principal of the Cauchy transform $\mathcal{C} \mu$ at $z \in \mathbb{C}$ is

$$
\text { p.v.C } \mu(z)=\lim _{\varepsilon \rightarrow 0} \mathcal{C}_{\varepsilon} \mu(z),
$$

whenever the limit exists. The maximal Cauchy transform of $\mu$ is

$$
\mathcal{C}_{*} \mu(z)=\sup _{\varepsilon>0}\left|\int_{|\xi-z|>\varepsilon} \frac{1}{\xi-z} d \mu(\xi)\right| .
$$

Obviously, the existence of p.v.C $\mu(z)$ implies that $\mathcal{C}_{*} \mu(z)$ is finite. The converse needs not to be true.

Recall also that the upper and lower linear densities of $\mu$ at $z$ are defined, respectively, by

$$
\Theta_{\mu}^{*}(z)=\limsup _{r \rightarrow 0} \frac{\mu(B(z, r))}{2 r}, \quad \Theta_{*, \mu}(z)=\liminf _{r \rightarrow 0} \frac{\mu(B(z, r))}{2 r} .
$$

When both densities coincide, one writes $\Theta_{\mu}(z):=\Theta_{\mu}^{*}(z)=\Theta_{*, \mu}(z)$, and one calls it linear density. A Dirac delta on a point $z \in \mathbb{C}$ is denoted by $\delta_{z}$.

Mattila and Melnikov proved in [MMe] that if $E \subset \mathbb{C}$ has finite length and is rectifiable, then p.v.C $\mu(z)$ exists $\mathcal{H}^{1}$-a.e. on $E$, for any Borel measure $\mu$ (see [Ve1] for an easier proof). Using this result and Léger's Theorem 2.5 it was shown later in [To3] that if $\sigma$ is a measure with linear growth such that the Cauchy transform is bounded on $L^{2}(\sigma)$, then p.v.C $\mu(z)$ exists $\sigma$-a.e. As a consequence, by Theorem 2.9, for any Borel measure $\mu$ on $\mathbb{C}$, p.v.C $\mu(z)$ exists $\gamma$-a.e., that is to say, the set where p.v.C $\mu(z)$ fails to exist has zero analytic capacity (this result answers a question from Verdera [Ve2]).

In the converse direction (existence of principal values implies rectifiability) the first result was obtained by Mattila [Ma3]: he proved that if $\Theta_{*, \mu}(z)>0$ and p.v. $\mathcal{C} \mu(z)$ exists (and is finite) for $\mu$-a.e. $z \in \mathbb{C}$, then $\mu$ is concentrated on a rectifiable set, that is $\mu$ vanishes out of a rectifiable set. Let us remark that this 
result was obtained before the proof of the identity (4) which relates the Cauchy transform and curvature. Mattila's techniques were based on the use of tangent measures. In [Huo], Huovinen extended Mattila's result to other kernels in the plane different from the Cauchy transform.

In [Ma3] Mattila wondered if the assumption $\Theta_{*, \mu}(z)>0 \mu$-a.e. might be replaced by $\Theta_{\mu}^{*}(z)>0 \mu$-a.e. In [To2] a partial answer was given: using the $T(b)$ theorem of Nazarov, Treil and Volberg in [NTV2] and the "curvature method" (identity (4) and Léger's Theorem 2.5 were used), it was shown that the main result in [Ma3] also holds for measures such that $0<\Theta_{\mu}^{*}(z)<\infty \mu$-a.e. Also, it was proved that one can replace the assumption on the existence of principal values by finiteness of the maximal Cauchy transform. As a consequence, one deduces that if $E \subset \mathbb{C}$ has finite length and $\mathcal{C}_{*} \mathcal{H}_{\mid E}^{1}(z)<\infty \mathcal{H}^{1}$-a.e. on $E$, then $E$ must be rectifiable.

A complete answer to Mattila's question has been given in [To10] recently. The precise result is the following.

Theorem 3.1. Let $\mu$ be a finite Radon measure on the complex plane and set $E=$ $\left\{z \in \operatorname{supp}(\mu): \mathcal{C}_{*} \mu(z)<\infty\right\}$. Then $\mu_{\mid E}$ can be decomposed as $\mu_{\mid E}=\mu_{d}+\mu_{r}+\mu_{0}$, where

$$
\mu_{d}=\sum_{i} a_{i} \delta_{z_{i}}
$$

for some $a_{i}>0$ and $z_{i} \in \mathbb{C}$,

$$
\mu_{r}=\sum_{i} g_{i} \mathcal{H}_{\mid \Gamma_{i}}^{1}
$$

for some rectifiable curves $\Gamma_{i}$ and non negative functions $g_{i} \in L^{1}\left(\mathcal{H}_{\mid \Gamma_{i}}^{1}\right)$, and

$$
\mu_{0}=\sum_{i} \sigma_{i}
$$

where $\sigma_{i}$ are measures with finite curvature such that $\Theta_{\sigma_{i}}(z)=0$ for $\sigma_{i}$-a.e. $z \in \mathbb{C}$.

Notice that the preceding result asserts that there is a kind of "dimensional gap" between 0 and 1 for the measures $\mu$ such that $\mathcal{C}_{*} \mu(z)<\infty \mu$-a.e. For instance, for $0<s<1$ there are no measures of the form $\mu=\mathcal{H}_{\mid E}^{s}$, with $0<\mathcal{H}^{s}(E)<\infty$, such that $\mathcal{C}_{*} \mu(z)<\infty \mu$-a.e.

A straightforward corollary of the theorem above is the following.

Corollary 3.2. Let $\mu$ be a finite Radon measure on the complex plane such that $\Theta_{\mu}^{*}(z)>0$ for $\mu$-a.e. $z \in \mathbb{C}$. If $\mathcal{C}_{*} \mu(z)<\infty$ at $\mu$-a.e. $z \in \mathbb{C}$, then $\mu$ can be decomposed as $\mu=\mu_{d}+\mu_{r}$, where $\mu_{d}=\sum_{i} a_{i} \delta_{z_{i}}$ for some $a_{i}>0$ and $z_{i} \in \mathbb{C}$, and $\mu_{r}=\sum_{i} g_{i} \mathcal{H}_{\mid \Gamma_{i}}^{1}$ for some rectifiable curves $\Gamma_{i}$ and non negative functions $g_{i} \in L^{1}\left(\mathcal{H}_{\mid \Gamma_{i}}^{1}\right)$.

In particular, under the assumptions of the corollary, $\mu$ is concentrated on a countable union of rectifiable curves.

The main difficulty to prove Theorem 3.1 consists in proving that if $\mathcal{C}_{*} \mu(z)<\infty$ $\mu$-a.e. on $\mathbb{C}$, then on the set $\left\{z: \Theta_{\mu}^{*}(z)=\infty\right\} \mu$ must be discrete (i.e. the addition 
of countably many point masses). Once this is proved, one can argue as in [To2]. A basic tool for the proof is the identity (4) again. However, notice that (4) holds for measures with linear growth, and the measures $\mu$ considered in Theorem 3.1 may be be very far from having this property. This is the main obstacle that is overcome in [To10].

Before [To10], Jones and Poltoratski proved in [JP], among other things, that if $\mu$ is supported on a line (and more generally on a $\mathcal{C}^{1}$ curve) and $\mathcal{C}_{*} \mu(z)<\infty \mu$-a.e., then $\mu$ equals a countable collection of point masses plus some measure absolutely continuous with respect to arc length. Observe that this result is implied by Corollary 3.2 , because any measure $\mu$ supported on a line satisfies $\Theta_{\mu}^{*}(z)>0 \mu$-a.e. In $[\mathrm{JP}]$ it was also shown that if one does not assume $\mu$ to be supported on a line and instead one asks the same conditions as Mattila in [Ma3] (i.e. $\Theta_{*, \mu}(z)>0$ and p.v.C $\mu(z)$ exists $\mu$ a.e. on $\mathbb{C})$, then the conclusion of Corollary 3.2 holds: $\mu$ equals a countable collection of point masses plus some measure absolutely continuous with respect to arc length on a rectifiable set.

The same techniques used for Theorem 3.1 also yield the following result, proved previously by Jones and Poltoratski [JP] when $\mu$ is supported on a $\mathcal{C}^{1}$ curve.

Theorem 3.3. Let $\mu$ be a finite Radon measure on the complex plane and set $E=\left\{x \in \operatorname{supp}(\mu): \mathcal{C}_{\varepsilon} \mu(x)=o(\mu(B(x, \varepsilon) / \varepsilon)\right.$ as $\varepsilon \rightarrow 0+\}$. Then $\mu_{\mid E}$ can be decomposed as $\mu_{\mid E}=\mu_{d}+\mu_{r}+\mu_{0}$, with $\mu_{d}$, $\mu_{r}$, and $\mu_{0}$ as in Theorem 3.1.

The arguments in [To10] rely on the relationship between the Cauchy transform and curvature and so they do not extend to higher dimensions (i.e. to Riesz transforms). This is not the case with the results in [Ma3] and [JP]. Mattila's results in [Ma3] have been extended to the case of Riesz transforms by Mattila and Preiss $[\mathrm{MPr}]$, while some theorems in [JP] deal both with Cauchy and Riesz transforms. See next section for the precise definition of Riesz transforms.

\section{Lipschitz harmonic capacity and Riesz transforms}

Let $E \subset \mathbb{R}^{d}$ be a compact set. Its Lipschitz harmonic capacity is

$$
\kappa(E)=\sup |\langle\Delta f, 1\rangle|,
$$

where $\Delta$ stands for the Laplacian in the distributional sense and the supremum is taken over all functions $f$ which are harmonic in $\mathbb{R}^{d} \backslash E$ and Lipschitz on $\mathbb{R}^{d}$, with $\|\nabla f\|_{\infty} \leq 1$ and $\nabla f(\infty)=0$. If, in addition, in the supremum above one asks $f$ to be $\mathcal{C}^{1}$, then one obtains the so called $\mathcal{C}^{1}$ harmonic capacity.

Given a distribution $\nu$ on $\mathbb{R}^{d}$, let $R(\nu)=\frac{y}{|y|^{d}} * \nu$ be its (vectorial) Riesz transform, so that when $\nu$ is a real measure,

$$
R \nu(x)=\left(\frac{y}{|y|^{d}} * \nu\right)(x)=\int \frac{x-y}{|x-y|^{d}} d \nu(y) .
$$

If $\mu$ is a positive Borel measure and $f$ a $\mu$-measurable function, we consider the operator $R_{\mu} f:=R(f d \mu)$. For $\varepsilon>0$, the truncated Riesz transforms $R_{\varepsilon}, R_{\mu, \varepsilon}$ are 
defined analogously to $\mathcal{C}_{\varepsilon}$ and $\mathcal{C}_{\mu, \varepsilon}$. One says that $R_{\mu}$ is bounded on $L^{2}(\mu)$ if the operators $R_{\mu, \varepsilon}$ are bounded on $L^{2}(\mu)$ uniformly on $\varepsilon>0$.

An equivalent definition of $\kappa(E)$ in terms of Riesz transforms is the following:

$$
\kappa(E)=\sup \sigma_{d}^{-1}|\langle\nu, 1\rangle|,
$$

where $\sigma_{d}$ stands for the $(d-1)$-dimensional volume of the sphere $\{|x|=1\}$ in $\mathbb{R}^{d}$ and the supremum is taken over all real distributions $\nu$ supported on $E$ such that $R \nu$ is a bounded function on $\mathbb{R}^{d}$, with $\|R \nu\|_{\infty} \leq 1$. An analogous definition exists for the $\mathcal{C}^{1}$ harmonic capacity.

The notions of Lipschitz harmonic capacity and $\mathcal{C}^{1}$ harmonic capacity were introduced by Paramonov [Par] in order to study problems of approximation of harmonic functions in the $\mathcal{C}^{1}$ norm. These capacities can be considered as real versions of the analytic capacity $\gamma$ and the continuous analytic capacity $\alpha$ in $\mathbb{R}^{d}$, $d \geq 2$, respectively. Indeed, in $\mathbb{R}^{2}, \kappa$ coincides (modulo a multiplicative absolute constant) with the so called real analytic capacity $\gamma_{\mathbb{R}}$ :

$$
\gamma_{\mathbb{R}}(E)=\sup \left|f^{\prime}(\infty)\right|
$$

where the supremum is taken over all functions $f$ analytic on $\mathbb{C} \backslash E$ with $\|f\|_{\infty} \leq 1$ which are Cauchy transforms of real distributions. Analogously with respect to the $\mathcal{C}^{1}$ harmonic capacity.

Some geometric properties of $\kappa$ in $\mathbb{R}^{d}$ have been studied in [MPa]. In particular, the relationship with the $(d-1)$-dimensional Hausdorff measure. Analogously to the case of analytic capacity, $(d-1)$-dimensional rectifiability seems to play a key role in the understanding of $\kappa$. Recall that $E \subset \mathbb{R}^{d}$ is called $n$-rectifiable if there are Lipschitz maps $f_{i}: \mathbb{R}^{n} \rightarrow \mathbb{R}^{d}$ such that

$$
\mathcal{H}^{n}\left(E \backslash \bigcup_{i=1}^{\infty} f_{i}\left(\mathbb{R}^{n}\right)\right)=0
$$

For example, if $\mathcal{H}^{d-1}(E)>0$ and $E$ is $(d-1)$-rectifiable, then $\kappa(E)>0$. However, unlike in the case of analytic capacity, it is not known if the compact sets $E \subset \mathbb{R}^{d}$ with $\mathcal{H}^{d-1}(E)<\infty$ and $\kappa(E)=0$ must be purely $(d-1)$-unrectifiable (recall Theorem 2.6). The main obstacle is the lack of a formula similar to (4) which relates the $L^{2}$ norm of the Riesz transforms with something which has some geometric meaning, or at least with something non negative, which may act as a square function for Riesz transforms. See [Fa] for this question.

Because of the same reason, many of the results in connection with the Cauchy transform which have been described in Sections 2 and 3 are missing in the case of Riesz transforms. See next section for some open questions.

On the other hand, although in the proof of the comparability between $\gamma$ and $\gamma_{+}$in [To5], curvature seems to play an important role, Volberg [Vo] has been able to show $\kappa$ and $\kappa_{+}$are also comparable $\left(\kappa_{+}\right.$is defined like $\kappa$ in (11), but taking only the supremum over positive measures $\nu$ supported on $E$ ). As a consequence, $\kappa$ is countably semiadditive, because $\kappa_{+}$has the following characterization (compare 
with Theorem 2.7):

$\kappa_{+}(E) \approx \sup \left\{\mu(E): \operatorname{supp}(\mu) \subset E, \mu(B(x, r)) \leq r^{d-1} \forall x, r,\left\|R_{\mu}\right\|_{L^{2}(\mu), L^{2}(\mu)} \leq 1\right\}$,

which is clearly subadditive. The main difference between the arguments used in [To5] for analytic capacity and ones in [Vo] for Lipschitz harmonic capacity stems from the choice of an appropriate potential useful for $\kappa_{+}$which is very different from the one used for $\gamma_{+}$in [To5].

Another paper concerning Lipschitz harmonic capacity is [MT]. In this article some Cantor sets in $\mathbb{R}^{d}$ are considered, and their Lipschitz harmonic capacity is estimated. The results are analogous to the ones in [MTV] for analytic capacity. The main difficulty in [MT] consists in estimating the $L^{2}$ norm of the Riesz transforms with respect to the natural probability measure associated to these Cantor sets.

\section{Some open problems}

In this section we collect some open problems related to analytic capacity, the Cauchy and Riesz transforms, and rectifiability. Most of them are well known, and there is no attempt at originality or completeness.

1. Subadditivity of analytic capacity. Is analytic capacity subadditive? That is to say, we are asking if $\gamma$ is semiadditive with constant 1:

$$
\gamma(E \cup F) \leq \gamma(E)+\gamma(F), \quad \text { for all compact sets } E, F \subset \mathbb{C} .
$$

There are a couple of facts that suggest that this may be true: first, $\gamma$ is countably semiadditive; and second, the above inequality holds when $E$ and $F$ are disjoint compact connected sets $[\mathrm{Su}]$.

Even when $\gamma(F)=0$, the identity $\gamma(E \cup F)=\gamma(E)$ remains unproved.

2. The Cauchy capacity. Given $E \subset \mathbb{C}$ compact, consider the so called Cauchy capacity of $E$ :

$$
\gamma_{C}(E)=\sup |\nu(E)|,
$$

where the supremum is taken over all complex measures supported on $E$ such that $|\mathcal{C} \nu(z)| \leq 1$ for $z \notin E$. This definition is similar to the one of analytic capacity, but now the supremum is restricted to Cauchy transforms of complex measures instead of distributions. Is it true that

$$
\gamma(E)=\gamma_{C}(E) ?
$$

Notice that Theorem 2.9 implies that $\gamma(E) \approx \gamma_{C}(E)$. However, it is not known if the identity holds, except in some particular cases: for example, when $E$ has finite length.

There exist compact sets $E$ and functions $f$ that are bounded and analytic on $\mathbb{C} \backslash E$ which are not the Cauchy transform of any complex measure $\nu$. Nevertheless, the identity $\gamma(E)=\gamma_{C}(E)$ may still hold. See [Kha1] and [Kha2] for more information about this question. 
3. Analytic capacity and Favard length. Recall the definition of Favard length in (7). We have already mentioned in Subsection 2.4 that Vitushkin's conjecture fails for sets with infinite length [Ma1]. In fact, Jones and Murai [JM] showed that there are sets with zero Favard length and positive analytic capacity.

So one of the implications in Vitushkin's conjecture is false. However the other implication is still open:

$$
\operatorname{Fav}(E)>0 \quad \Rightarrow \quad \gamma(E)>0 ?
$$

Observe that by the characterization of $\gamma$ in terms of curvature, this question can be restated in a more geometric way.

A related problem is the following. Let $E$ be the so called corner quarters Cantor set. This set is constructed as follows. Let $Q^{0}=[0,1] \times[0,1]$. At the first step we take four closed squares inside $Q^{0}$ with side length $1 / 4$, with sides parallel to the coordinate axes, and so that each square contains a vertex of $Q^{0}$. At the second step we apply the preceding procedure to each of the four squares obtained in the first step, so that we get 16 squares of side length $1 / 16$. Proceeding inductively, at each step we obtain $4^{n}$ squares $Q_{j}^{n}, j=1, \ldots, 4^{n}$ with side length $4^{-n}$. We denote

$$
E_{n}=\bigcup_{j=1}^{4^{n}} Q_{j}^{n}
$$

and we define $E=\bigcap_{n=1}^{\infty} E_{n}$. This set has positive finite length and is purely unrectifiable, and so it has zero analytic capacity by David's theorem (this had been proved before David's result in [Gar1] and [Iv]). In fact, by [MTV] one has the asymptotic estimate $\gamma\left(E_{n}\right) \approx n^{-1 / 2}$. By Besicovitch theorem, we have $\operatorname{Fav}(E)=0$. However, the asymptotic behavior of $\operatorname{Fav}\left(E_{n}\right)$ as $n \rightarrow \infty$ is not known. An interesting problem consists in finding more or less precise estimates for the asymptotic behavior of $\operatorname{Fav}\left(E_{n}\right)$.

From some results due to Mattila [Ma2] one gets the lower estimate $\operatorname{Fav}\left(E_{n}\right) \gtrsim$ $1 / n$. Other recent results by Peres and Solomyak [PS] for random Cantor sets suggest that the estimate $\operatorname{Fav}\left(E_{n}\right) \approx 1 / n$ may hold. However, up to now the best upper estimate is

$$
\operatorname{Fav}\left(E_{n}\right) \lesssim \exp \left(-a \log _{*} n\right)
$$

where $C, a$ are positive absolute constants and $\log _{*}$ is the function

$$
\log _{*} x=\min \{n \in \mathbb{N}: \underbrace{\log \log \cdots \log }_{n} x \leq 1\} .
$$

Inequality (12) has been obtained in [PS]. It turns out that $\exp \left(-a \log _{*} n\right) \rightarrow 0$ extremely slowly, much more slowly than $1 / n$.

Many difficulties that arise in connection with Favard length are related to the fact that there is no a quantitative proof of Besicovitch theorem. For more information concerning Favard length and projections we recommend to have a look at the nice survey [Ma4]. 
4. Vanishing Cauchy transforms. Let $\nu$ be a complex Borel measure on $\mathbb{C}$. Suppose that p.v.C $\nu(z)$ exists and vanishes $|\nu|$-a.e. on $\mathbb{C}$. Does this imply that $\nu$ is an atomic measure?

Notice that if $\nu$ is a positive measure, then Theorem 3.1 applies to $\nu$ and so in this case we know that $\nu=\nu_{d}+\nu_{r}+\nu_{0}$, where $\nu_{d}$ is discrete and $\nu_{r}, \nu_{0}$ are as in Theorem 3.1.

In [TVe] two particular cases have been studied. In the first one $\nu$ is absolutely continuous with respect to Lebesgue measure, and in the second one $\nu$ is a positive measure with linear growth and finite curvature. In both cases, if p.v.C $\mathcal{C}(z)$ exists and vanishes $|\nu|$-a.e., then $\nu=0$.

Let us remark that there are positive discrete (non zero) measures such that p.v.C $\nu(z)$ exists and vanishes $\nu$-a.e. A trivial example is a single point mass. One can also construct other examples with countably many point masses.

5. Riesz transforms and rectifiability. Let $E \subset \mathbb{R}^{d}$ be a compact set with $0<\mathcal{H}^{n}(E)<\infty$, for some integer $0<n<d$. Take $\mu=\mathcal{H}_{\mid E}^{n}$ and consider the $n$-dimensional Riesz transform:

$$
R_{\mu}^{n} f(x)=\int \frac{x-y}{|x-y|^{n+1}} f(y) d \mu(y),
$$

for $f \in L^{2}(\mu)$, and $x \notin E$. As usual, we say that $R_{\mu}^{n}$ is bounded in $L^{2}(\mu)$ if the corresponding $\varepsilon$-truncated operators are bounded in $L^{2}(\mu)$ uniformly on $\varepsilon>0$. If $R_{\mu}^{n}$ is bounded in $L^{2}(\mu)$, is then $E n$-rectifiable? The answer is known (and it is positive in this case) only for $n=1$, because the curvature method works for $n=1$. By [Vo], when $n=d-1$ this question is equivalent to the following, which has already appeared in Section 4: is it true that $\kappa(E)=0$ if and only if $E$ is purely $(d-1)$-unrectifiable?

A variant of this problem consist in taking $E \mathrm{AD}$ regular and $n$-dimensional. If $R_{\mu}^{n}$ is bounded in $L^{2}(\mu)$, is then $E$ uniformly $n$-rectifiable? For the definition of uniform rectifiability, see [DS1] and [DS2] (for the reader's convenience let us say that, roughly speaking, uniform rectifiability is the same as rectifiability plus some quantitative estimates). For $n=1$ the answer is true again, because of curvature. The result is from Mattila, Melnikov and Verdera [MMV]. For $n>1$, in [DS1] and [DS2] some partial answers are given. Let $H_{n}$ be class of all the operators $T$ defined as follows:

$$
T f(x)=\int k(x-y) f(y) d \mu(x),
$$

where $k$ is some odd kernel (i.e. $k(-x)=-k(x)$ ) smooth away from the origin such that $|x|^{n+j}\left|\nabla^{j} k(x)\right| \in L^{\infty}\left(\mathbb{R}^{d} \backslash\{0\}\right)$ for $j \geq 0$. Suppose that all operators $T$ from $H_{n}$ are bounded in $L^{2}(\mu)$. Then it is shown in [DS1] that $E$ is uniformly rectifiable. See [DS2] for other related results.

Consider again the case of a general compact set $E \subset \mathbb{R}^{d}$ with $0<\mathcal{H}^{n}(E)<\infty$, for some integer $0<n<d$, and set $\mu=\mathcal{H}_{\mid E}^{n}$. If $\mu$ satisfies

$$
\liminf _{r \rightarrow 0} \frac{\mu(B(x, r))}{r^{n}}>0 \quad \mu \text {-a.e. on } \mathbb{R}^{d}
$$


then the $\mu$-a.e. existence of the principal value $\lim _{\varepsilon \rightarrow 0} R_{\mu, \varepsilon}^{n} 1(x)$ implies that $E$ is $n$-rectifiable, by a theorem of Mattila and Preiss [MPr]. However, this does not help to solve the questions above because it is not known if the $L^{2}(\mu)$ boundedness of the Riesz transforms $R_{\mu}^{n}$ implies the existence of principal values. Notice the contrast with the case of the Cauchy transform (see Section 3), where the latter assertion is known to be true, because of curvature again.

I would like to thank Joan Verdera for his remarks on preliminary versions of this paper.

\section{References}

[Ah] L. V. Ahlfors, Bounded analytic functions, Duke Math. J. 14 (1947). 1-11.

[Ch] M. Christ, A T (b) theorem with remarks on analytic capacity and the Cauchy integral, Colloq. Math. 60/61 (1990), no. 2, 601-628.

[CMM] R.R. Coifman, A. McIntosh and Y. Meyer, L'integrale de Cauchy définit un opérateur borné sur $L^{2}$ pour les courbes lipschitziennes, Ann. of Math. (2)116 (1982), 361-387.

[Da] G. David, Unrectifiable 1-sets have vanishing analytic capacity, Revista Mat. Iberoamericana 14(2) (1998), 369-479.

[DM] G. David and P. Mattila, Removable sets for Lipschitz harmonic functions in the plane, Rev. Mat. Iberoamericana 16 (2000), no. 1, 137-215.

[DS1] G. David and S. Semmes, Singular integrals and rectifiable sets in $R_{n}$ : Au-delà des graphes lipschitziens, Astérisque No. 193 (1991).

[DS2] G. David and S. Semmes. Analysis of and on uniformly rectifiable sets. Mathematical Surveys and Monographs, 38. American Mathematical Society, Providence, RI, 1993.

[DØ] A. M. Davie and B. Øksendal, Analytic capacity and differentiability properties of finely harmonic functions, Acta Math. 149 (1982), no. 1-2, 127-152.

[Fa] H. Farag, The Riesz kernels do not give rise to higher-dimensional analogues of the Menger-Melnikov curvature, Publ. Mat. 43 (1999), no. 1, 251-260.

[FFP] F. Ferrari, B. Franchi and H. Pajot, The Geometric Traveling Salesman Problem in the Heisenberg Group, Preprint (2005).

[Gam] T. Gamelin, Uniform Algebras, Prentice Hall, Englewood Cliffs N.J., 1969.

[Gar1] J.B. Garnett, Positive length but zero analytic capacity, Proc. Amer. Math. Soc. 24 (1970), 696-699.

[Gar2] J. Garnett, Analytic capacity and measure, Lecture Notes in Math. 297, Springer-Verlag, 1972.

[Hah] I. Hahlomaa, Menger curvature and Lipschitz parametrizations in metric spaces, Fund. Math. 185 (2005), no. 2, 143-169. 
[Huo] P. Huovinen, Singular integrals and rectifiability of measures in the plane. Dissertation, University of Jyvskyl, Jyvskyl, 1997. Ann. Acad. Sci. Fenn. Math. Diss. No. 109 (1997), 63 pp.

[Iv] L.D. Ivanov, On sets of analytic capacity zero, in "Linear and Complex Analysis Problem Book 3" (part II), Lectures Notes in Mathematics 1574, SpringerVerlag, Berlin, 1994, pp. 150-153.

[GV] J. Garnett and J. Verdera, Analytic capacity, bilipschitz maps and Cantor sets, Math. Res. Lett. 10 (2003), 515-522.

[Jo] P.W. Jones, Rectifiable sets and the traveling salesman problem, Invent. Math. 102 (1990), 1-15.

[JM] P.W. Jones and T. Murai, Positive analytic capacity but zero Buffon needle probability, Pacific J. Math. 133 (1988), 89-114.

[JP] P.W. Jones and A.G. Poltoratski, Asymptotic growth of Cauchy transforms, Ann. Acad. Sci. Fenn. Math. 29 (2004), no. 1, 99-120.

[JyM] H. Joyce and P. Mörters. A set with finite curvature and projections of zero length. J. Math. Anal. Appl. 247 (2000), 126-135.

[Kha1] S. Ya. Khavinson, Golubev sums: a theory of extremal problems that are of the analytic capacity problem type and of accompanying approximation processes. (Russian) Uspekhi Mat. Nauk 54 (1999), no. 4(328), 75-142; translation in Russian Math. Surveys 54 (1999), no. 4, 753-818.

[Kha2] S. Ya. Khavinson, Duality relations in the theory of analytic capacity. (Russian) Algebra i Analiz 15 (2003), no. 1, 3-62; translation in St. Petersburg Math. J. 15 (2004), no. 1, 1-40.

[Lé] J.C. Léger, Menger curvature and rectifiability, Ann. of Math. 149 (1999), 831869.

[Lr] G. Lerman, Quantifying curvelike structures of measures by using $L^{2}$ Jones quantities, Comm. Pure Appl. Math. 56 (2003), 1294-1365.

[MPV] J. Mateu, L. Prat and J. Verdera, The capacities associated to signed Riesz kernels, and Wolff potentials, J. Reine Angew. Math. 578 (2005), 201-223.

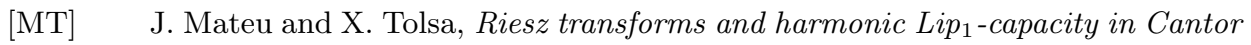
sets, Proc. London Math. Soc. 89(3) (2004), 676-696.

[MTV] J. Mateu, X. Tolsa and J. Verdera, The planar Cantor sets of zero analytic capacity and the local T(b)-Theorem, J. Amer. Math. Soc. 16 (2003), 19-28.

[Ma1] P. Mattila, Smooth maps, null sets for integralgeometric measure and analytic capacity, Ann. of Math. 123 (1986), 303-309.

[Ma2] P. Mattila, Orthogonal projections, Riesz capacities, and Minkowski content, Indiana Univ. Math. J. 39 (1990), no. 1, 185-198.

[Ma3] P. Mattila, Cauchy Singular Integrals and Rectifiability of Measures in the Plane, Adv. Math. 115 (1995), 1-34.

[Ma4] P. Mattila, Hausdorff dimension, projections, and Fourier transform, Publ. Mat. 48 (2004), 3-48.

[MMe] P. Mattila and M.S. Melnikov, Existence and weak type inequalities for Cauchy integrals of general measures on rectifiable curves and sets, Proc. Amer. Math. 120(1994), 143-149. 
[MMV] P. Mattila, M.S. Melnikov and J. Verdera, The Cauchy integral, analytic capacity, and uniform rectifiability, Ann. of Math. (2) 144 (1996), 127-136.

[MPa] P. Mattila and P.V. Paramonov, On geometric properties of harmonic Lip - $^{-}$ capacity, Pacific J. Math. 171:2 (1995), 469-490.

[MPr] P. Mattila and D. Preiss, Rectifiable measures in $\mathbb{R}^{n}$ and existence of principal values for singular integrals, J. London Math. Soc. (2) 52 (1995), no. 3, 482496.

[Me] M. S. Melnikov, Analytic capacity: discrete approach and curvature of a measure, Sbornik: Mathematics 186(6) (1995), 827-846.

$[\mathrm{MeV}] \quad$ M. S. Melnikov and J. Verdera, A geometric proof of the $L^{2}$ boundedness of the Cauchy integral on Lipschitz graphs, Internat. Math. Res. Notices (1995), 325-331.

[NTV1] F. Nazarov, S. Treil and A. Volberg, Cauchy integral and Calderón-Zygmund operators in non-homogeneous spaces, Internat. Res. Math. Notices 15 (1997), 703-726.

[NTV2] F. Nazarov, S. Treil and A. Volberg, The T(b) theorem on non-homogeneous spaces that proves a conjecture of Vitushkin, Preprint n. 519, Centre de Recerca Matemàtica, Barcelona (2002).

[Ok] K. Okikiolu, Characterization of subsets of rectifiable curves in $R^{n}$, J. London Math. Soc. (2) 46 (1992), 336-348.

[Pa] H. Pajot, Analytic capacity, rectifiability, Menger curvature and the Cauchy integral, Lecture Notes in Math. 1799 (2002), Springer.

[Par] P.V. Paramonov, On harmonic approximation in the $\mathcal{C}^{1}$ norm, (Russian) Mat. Sb. 181 (1990), no. 10, 1341-1365; translation in Math. USSR-Sb. 71 (1992), no. 1, 183-207.

[PS] Y. Peres and B. Solomyak, How likely is Buffon's needle to fall near a planar Cantor set?, Pacific J. Math. 204 (2002), no. 2, 473-496.

[Pr] L. Prat, Potential theory of signed Riesz kernels: capacity and Hausdorff measures, Int. Math. Res. Notices 19 (2004), 937-981.

[Sch] R. Schul, Characterization of Subsets of Rectifiable Curves in Hilbert Space and the Analyst's Traveling Salesman Problem, Ph. Thesis, Yale (2005).

[Su] N. Suita, On subadditivity of analytic capacity for two continua, Kodai Math. J. 7 (1984), 73-75.

[To1] X. Tolsa, $L^{2}$-boundedness of the Cauchy integral operator for continuous measures, Duke Math. J. 98(2) (1999), 269-304.

[To2] X. Tolsa, Principal values for the Cauchy integral and rectifiability. Proc. Amer. Math. Soc. 128(7) (2000), 2111-2119.

[To3] X. Tolsa, Cotlar's inequality and existence of principal values for the Cauchy integral without the doubling condition, J. Reine Angew. Math. 502 (1998), 199-235.

[To4] X. Tolsa, On the analytic capacity $\gamma_{+}$, Indiana Univ. Math. J. 51(2) (2002), 317-344.

[To5] X. Tolsa, Painlevé's problem and the semiadditivity of analytic capacity, Acta Math. 190:1 (2003), 105-149. 
[To6] X. Tolsa, The semiadditivity of continuous analytic capacity and the inner boundary conjecture, Amer. J. Math. 126 (2004), 523-567.

[To7] X. Tolsa, Bilipschitz maps, analytic capacity, and the Cauchy integral, Ann. of Math. 162:3 (2005), 1241-1302.

[To8] X. Tolsa, $L^{2}$ boundedness of the Cauchy transform implies $L^{2}$ boundedness of all Calderón-Zygmund operators associated to odd kernels, Publ. Mat. 48 (2) (2004), 445-479.

[To9] X. Tolsa, Finite curvature of arc length measure implies rectifiability: a new proof, Indiana Univ. Math. J. 54 (2005), no. 4, 1075-1105.

[To10] X. Tolsa, Growth estimates for Cauchy integrals of measures and rectifiability, Preprint (2005). To appear in Geom. Funct. Anal.

[TVe] X. Tolsa and J. Verdera, May the Cauchy transform of a non-trivial finite measure vanish on the support of the measure?, Preprint (2005). To appear in Ann. Acad. Sci. Fenn. Math.

[Uy] Nguyen Xuan Uy, Removable sets of analytic functions satisfying a Lipschitz condition, Ark. Mat. 17 (1979), 19-27.

[Ve1] J. Verdera, A weak type inequality for Cauchy transforms of finite measures, Publ. Mat. 36 (1992), 1029-1034.

[Ve2] J. Verdera, Removability, capacity and approximation. In "Complex Potential Theory", (Montreal, PQ, 1993), NATO Adv. Sci. Int. Ser. C Math. Phys. Sci. 439, Kluwer Academic Publ., Dordrecht, 1994, pp. 419-473.

[Ve3] J. Verdera, On the T(1)-theorem for the Cauchy integral, Ark. Mat. 38 (2000), $183-199$

[Vi1] A. G. Vitushkin, Example of a set of positive length but of zero analytic capacity, (Russian) Dokl. Akad. Nauk SSSR 127 (1959), 246-249.

[Vi2] A. G. Vitushkin, Estimate of the Cauchy integral, (Russian) Mat. Sb. 71 (113) (1966), 515-534.

[Vi3] A. G. Vitushkin, The analytic capacity of sets in problems of approximation theory, Uspekhi Mat. Nauk. 22(6) (1967), 141-199 (Russian); in Russian Math. Surveys 22 (1967), 139-200.

[Vo] A. Volberg, Calderón-Zygmund capacities and operators on nonhomogeneous spaces. CBMS Regional Conf. Ser. in Math. 100, Amer. Math. Soc., Providence, 2003.

Institució Catalana de Recerca i Estudis Avançats (ICREA) and Departament de Matemàtiques, Universitat Autònoma de Barcelona, Spain

E-mail: xtolsa@mat.uab.es 